\title{
Des maîtres entretenus aux ingénieurs (1819-1971)
}

Jean André Berthiau

\section{OpenEdition}

Journals

Édition électronique

URL : https://journals.openedition.org/tc/1401

DOI : $10.4000 /$ tc. 1401

ISSN : 1952-420X

\section{Éditeur}

Éditions de l'EHESS

\section{Édition imprimée}

Date de publication : 1 juin 2005

ISSN : 0248-6016

\section{Référence électronique}

Jean André Berthiau, « Des maîtres entretenus aux ingénieurs (1819-1971) », Techniques \& Culture [En ligne], 45 | 2005, mis en ligne le 22 mai 2008, consulté le 29 septembre 2022. URL : http:// journals.openedition.org/tc/1401; DOI : https://doi.org/10.4000/tc.1401

Ce document a été généré automatiquement le 29 septembre 2022.

Tous droits réservés 


\title{
Des maîtres entretenus aux ingénieurs (1819-1971)
}

\author{
Jean André Berthiau
}

1 Les premiers constructeurs de la marine militaire, qualifiés de «maîtres charpentiers » dans l'ordonnance royale de 1689 -la grande ordonnance de Colbert-, sont des praticiens qui conservent jalousement les secrets de leur art, règles empiriques transmises à l'intérieur de réseaux familiaux dont ils acquièrent la maitrise par une longue pratique sur le terrain ${ }^{1}$. Une école des élèves constructeurs est créée en 1741, puis réorganisée en 1765 par une ordonnance qui homologue le titre d'ingénieur constructeur et constitue le corps qui deviendra en 1800 celui des ingénieurs du génie maritime, recrutés pour la plupart par l'école polytechnique depuis 1795. Ils sont secondés sur les chantiers par la "maistrance», terme désignant l'ensemble des maîtres et contremaîtres qui exercent l'autorité sur les ouvriers. La marine « entretient » des maîtres pour chaque corps de métier impliqué dans la construction et la maintenance de la flotte. Ils portent le titre de «maîtres entretenus » et leur solde est annuelle; on dirait aujourd'hui qu'ils jouissent d'un contrat à durée indéterminée. La situation du personnel ouvrier, qui englobe les contremaîtres et les ouvriers, était alors beaucoup plus précaire ${ }^{2}$.

2 Le décret du 25 janvier 1793 institue des écoles pour les apprentis dans les ports de Brest, Toulon, Rochefort et Lorient. Deux instituteurs entretenus aux frais de la République seront chargés de leur instruction. L'un leur apprendra à lire, à écrire et l'arithmétique, l'autre leur enseignera le dessin, le tracé des plans et la pratique de la construction des vaisseaux. Ces écoles ont très probablement fonctionné un certain temps, nous n'en avons la preuve que pour Brest et Toulon où elles furent réactivées en 1817 et 1818 après être tombées en désuétude durant plusieurs années. Des écoles d'enseignement mutuel sont créées en vue de donner les premières bases d'instruction aux apprentis, généralement illettrés à leur entrée dans l'arsenal.

1819-1868 - Un enseignement organisé

3 L'inspecteur général du génie maritime Pierre-Jacques-Nicolas Rolland est à l'origine de la mise en place d'une formation professionnelle organisée. Son rapport de tournée 
dans les arsenaux en 1818, "dans le but de remédier aux plaintes fondées qui s'élevaient de tous les ports contre le défaut d'instruction des maîtres, contremaîtres et aides chargés de diriger au travail les ouvriers des diverses professions relatives aux constructions navales ", servira au baron Portal, ministre de la marine, qui prescrira, par une circulaire du 17 août 1819 :

«[...] de former dans chacun des ports de Brest, Rochefort et Toulon, une école spéciale, dans laquelle un certain nombre de jeunes ouvriers destinés à la maistrance et choisis parmi les plus actifs et les plus intelligents, seraient exercés aux méthodes pratiques des arts relatifs à l'architecture navale, qui ne se conçoivent bien qu'à l'aide de connaissances théoriques ».

Cette circulaire peut être considérée comme l'acte de naissance des écoles de maistrance.

5 L'enseignement est réparti sur deux ans, avec une heure de cours de mathématiques (arithmétique, géométrie, trigonométrie) ou de dessin, le matin, une heure l'après-midi pour repasser les cours du matin, et travail à l'atelier ou au chantier le reste du temps. Douze élèves sont admis dans chacune des trois écoles (2/3 de charpentiers, 1/3 d'autres professions).

Quelques jeunes ouvriers sont également envoyés à Châlons-sur-Marne pour suivre les cours de l'école royale des arts et métiers, mais l'expérience restera très limitée; dixsept élèves seulement recevront cet enseignement entre 1819 et 1827. Les écoles de maistrance suffisent aux besoins du service, elles coûtent peu et donnent de meilleurs résultats.

7 Après une expérience de douze ans, l'ordonnance royale du 1er janvier 1833 fixe l'organisation de ces écoles de maistrance. L'amiral comte de Rigny, ministre de la Marine, rappelle que le but de ces écoles «qui ont justifié les espérances qu'on avait conçues de leur établissement » est de former «une maistrance habile qui influera puissamment sur l'économie et la bonne exécution des constructions navales ». Le programme est précisé : arithmétique, y compris usage pratique des logarithmes, éléments de géométrie, préliminaires de géométrie descriptive, éléments de statique et stabilité des corps flottants, application aux divers travaux, dessin linéaire, tenue de comptabilité des ateliers. Les élèves iront à l'école le matin et à l'atelier l'après-midi. Brest aura 24 élèves dont 4 de Lorient, 4 de Cherbourg et 2 de Saint-Servan, Rochefort aura 14 élèves dont 2 de Bayonne, et Toulon 14 élèves. Le corps des maîtres entretenus est réorganisé en $1834^{3}$, leur effectif est fixé à 234. Des disparités étant apparues dans les programmes et l'organisation des études, une règle uniforme est établie en $1851^{4}$ pour les trois écoles de maistrance ainsi que pour les écoles élémentaires établies dans tous les ports. Le niveau de l'enseignement est renforcé et des places dans les écoles de maistrance sont réservées aux ouvriers des établissements d'Indret et de Guérigny. Saint-Servan et Bayonne ont alors cessé toute activité de construction. La durée des études est de deux ans: pendant la première année, les cours ont lieu le matin et l'atelier l'après-midi; la deuxième année, les élèves ont cours trois après-midi par semaine, le reste du temps, ils sont à l'atelier. Les effectifs sont maintenus dans chacune des trois écoles: Brest aura 24 élèves dont 4 de Lorient et 6 de Cherbourg, Rochefort 14 élèves dont 2 de Lorient, 3 d'Indret et 2 de Guérigny, et Toulon 14 élèves.

Pour les écoles élémentaires, les bases de l'instruction sont les devoirs de la religion, la lecture, l'écriture, la grammaire, les éléments du calcul et du dessin linéaire. La plupart 
des apprentis sont totalement illettrés à leur entrée à l'arsenal. Le mode d'enseignement se rapproche en principe de celui de l'enseignement mutuel.

Depuis les années 1820 , de très importants changements techniques sont intervenus dans les constructions navales : développement des machines à vapeur, remplacement des câbles en chanvre par des câbles-chaînes, progrès de l'artillerie avec les boulets explosifs, constructions en fer et apparition des cuirasses. On assiste ainsi entre 1851 et 1868 à un véritable bouleversement dans la composition de la flotte : la voile cède le pas à la vapeur avec des machines de plus en plus puissantes, l'hélice se substitue aux roues à aubes latérales.

10 Ces profondes modifications, tant techniques, entraînant la naissance de nouvelles professions, que sociales avec l'amélioration des statuts des personnels, nécessitent leur prise en compte dans l'organisation de l'enseignement.

11 Les carrières des personnels de la maistrance sont améliorées en 1865 avec la création du grade de maître principal et la possibilité d'accès de maîtres au corps du génie maritime $e^{5}$.

1868-1900 - L'enseignement à deux niveaux

12 Un enseignement à deux niveaux est institué en $1868^{6}$. Sept écoles préparatoires de maistrance sont établies dans les ports de Cherbourg, Brest, Lorient, Rochefort, Toulon et les établissements d'Indret et de Guérigny. L'enseignement dure 20 mois, avec une demi-journée à l'école et une demi-journée à l'atelier. Deux écoles normales de maistrance sont créées à Brest et à Toulon dans le but de «développer, par un enseignement supérieur, les connaissances déjà acquises ». L'enseignement dure 18 mois avec une demi-journée à l'école et une demi-journée à l'atelier. Les programmes d'enseignement sont très largement augmentés et développés de manière précise. C'est aussi l'époque du remplacement du fer par l'acier, qui constitue une nouvelle et très importante évolution dans les méthodes de construction des coques.

13 En 1877, la durée d'affectation dans les écoles est réduite, mais les élèves ont des cours à temps complet ${ }^{7}$. L'école de maistrance de Toulon est supprimée, celle de Brest devient école supérieure de maistrance, avec un cycle d'études de 13 mois, les écoles préparatoires des ports deviennent écoles de maistrance avec un cycle de 14 mois, celles des établissements d'Indret et Guérigny sont supprimées. Les écoles seront à nouveau réorganisées en $1881^{8}$. Cette dernière réorganisation concerne surtout le programme des examens d'admission.

14 Les ministres qui se sont succédé pendant ces soixante années ont bien eu le souci légitime d'élever le niveau des études afin de l'adapter aux besoins nouveaux des chantiers, mais la situation faite aux anciens élèves sur le plan de l'avancement et des rémunérations n'a pas suivi cette évolution. On observe de nombreux départs vers l'industrie, elle aussi en plein développement, ou vers d'autres services de l'État, que la marine cherche à éviter par des expédients. Ainsi des primes journalières sont-elles attribuées aux anciens élèves qui ont obtenu le certificat d'études ou le brevet de capacité 9 . Ces primes, bloquées à la caisse des dépôts et consignations, constituent un capital qui n'est remis à l'intéressé qu'au bout d'un certain nombre d'années de service. Cette mesure est restée en vigueur jusqu'en 1900.

15 À la fin du siècle, l'utilité des écoles d'apprentis a été très contestée et elles ont été progressivement supprimées. Elles seront réorganisées en 1919.

1900-1940 - Du brevet de capacité au diplôme d'ingénieur 

supérieures de la marine, qui remplace le brevet de capacité, délivré aux élèves ayant subi avec succès les épreuves de l'examen de sortie ${ }^{14}$. Cette consécration tant attendue de la valeur de l'enseignement dispensé est le fruit de nombreuses entrevues entre les représentants des personnels concernés -regroupés depuis 1921 au sein d'une amicale - et l'ensemble de la hiérarchie, au cours de congrès annuels à Paris. Cela a permis d'informer au plus haut niveau sur les réalités de la valeur des anciens élèves et de l'importance des fonctions qui leur étaient confiées.

1940-1949 - Naissance d'une grande école

ta seconde guerre mondiale, l'enseignement était dispensé dans quatre écoles techniques normales à Cherbourg, Brest, Lorient et Toulon -celle de Rochefort ayant disparu avec la fermeture de la direction des constructions navales de ce port en 1927et deux écoles techniques supérieures à Brest (constructions navales) et à Toulon (artillerie navale). Paradoxalement, il aura fallu la période particulièrement troublée des années d'occupation allemande pour que les écoles techniques trouvent enfin leur unité. La direction des industries navales, qui regroupait constructions navales et artillerie navale, a poursuivi, dans le calme de sa retraite vichyssoise, les réflexions engagées avant la guerre pour une refonte des programmes des écoles. L'existence de 
l'école technique supérieure de Toulon aura été très brève, elle disparaîtra en 1941 après avoir formé une centaine d'élèves. Les conséquences de la guerre entraînent la disparition des écoles de Cherbourg et de Lorient et le repli de celles de Brest à Indret de 1941 à 1943, puis leur regroupement avec celles de Toulon, à Aurillac de 1943 à 1949.

Contrairement à ce qui se passait dans les ports, les ingénieurs enseignants affectés à Aurillac n'ont plus que cette seule fonction. Ils ne sont plus accaparés par des responsabilités techniques de construction et des impératifs de délais qui les détournaient bien souvent de leurs fonctions d'enseignement. D'importantes refontes des programmes et du régime des écoles sont mises en place dès $1945^{15}$. Les élèves sont alors recrutés sur un concours commun, le premier tiers des candidats admis est affecté directement à l'école technique supérieure, dont les cours durent trois ans. Les suivants sont admis à l'école technique normale pour un cursus de 18 mois. À l'issue de ce cursus, les premiers classés à l'examen suivent un complément de cours pour une mise à niveau en mathématiques en vue de passer les épreuves de l'examen qui leur permettra de rejoindre l'école technique supérieure.

Les cours sont organisés de manière rationnelle. Les écoles d'apprentis dispensent un enseignement scolaire et une formation professionnelle en atelier sur trois ans, sanctionnés par un diplôme d'apprentissage «marine ", d'un niveau supérieur à celui du certificat d'aptitude professionnelle. Le jeune ouvrier, muni de son diplôme, doit alors travailler en atelier ou en chantier pendant au moins deux ans, avec la possibilité de suivre gratuitement des cours du soir. Ensuite, il peut suivre pendant un an à temps complet les cours d'une école préparatoire recrutant sur examen, afin d'acquérir les connaissances de mathématiques élémentaires qui lui permettront d'affronter le concours d'entrée aux écoles techniques. Les élèves de l'école technique supérieure devront au cours de la première année atteindre le niveau du certificat de mathématiques générales, et en seconde année ceux de mécanique rationnelle et de physique générale.

Ce système assure à ceux qui ne réussissent pas le concours d'entrée aux écoles techniques un complément de formation qui autorise le recrutement de base des niveaux intermédiaires de techniciens, dessinateurs, préparateurs, techniciens de laboratoire, etc.

1949-1971 - Les écoles à Brest-Pontanézen

26 À la rentrée scolaire de 1949, les écoles techniques des constructions et armes navales sont installées sur le site actuel de Pontanézen, quartier périphérique de Brest. Elles retrouvent une implantation maritime plus en rapport avec leur vocation. La promotion 1952 de l'école technique supérieure verra l'admission du premier candidat provenant des classes préparatoires des lycées (bachelier mathématiques et technique).

Après dix ans d'expérience, la sélection directe sur le classement du concours, adoptée en 1945, est remplacée en $1955^{16}$ par une sélection après un stage commun de cinq mois, que suivent tous les élèves admis au concours, et à l'issue duquel est établi un nouveau classement. Les premiers sont admis à l'école technique supérieure et les autres continuent leurs études à l'école technique normale. L'évolution des techniques entraîne de nouveaux problèmes de formation, dont certains sont résolus par l'envoi des élèves concernés dans des écoles de spécialisation; c'est le cas notamment de l'électronique et de l'aéronautique. Les excellents résultats généralement obtenus par les élèves dans ces écoles permettent d'affirmer la très bonne qualité de leur formation de base. 
28 En 1960, les anciens élèves connaîtront de nouvelles améliorations de carrière avec l'étape très importante de la nomination au grade d'ingénieur des directions de travaux à la sortie de l'école, pour les élèves ayant obtenu le diplôme d'ingénieur. Suivra en 1968 la réforme des corps de l'armement, qui leur ouvrira un plus large accès au corps de direction et enfin la mise en place d'une hiérarchie complète dans le corps des ingénieurs des études et techniques d'armement qui permet à d'anciens apprentis d'assumer avec succès des fonctions de direction et de donner ainsi toute la mesure de leurs capacités. C'est un magnifique exemple de promotion sociale. Après un siècle et demi d'existence, l'école technique supérieure des constructions navales est regroupée avec celles des armements terrestres et de l'aéronautique pour former l'école nationale supérieure des ingénieurs des études et techniques d'armement. La première promotion de l'ENSIETA a été recrutée en 1971. Les écoles d'apprentissage deviennent alors des écoles de formation technique et comprennent deux cycles distincts: les écoles de formation technique normales, destinées à la formation d'ouvriers qualifiés et les écoles de formation technique spéciales, conduisant aux concours d'admission au cours préparatoire IETA (préparation au concours ENSIETA) et à l'école technique normale. Ces écoles de formation technique ont aujourd'hui disparu.

Réflexions d'un ancien élève

Le baron Portal a toujours été considéré comme le fondateur des écoles de maistrance. S'il est incontestable que celui dont Tupinier disait que « c'était sans doute le ministre qui avait le mieux compris les problèmes de la marine » est bien le signataire de la circulaire de 1819 qui en consacre l'acte de naissance, notre étude a permis de dégager le rôle particulièrement important que jouèrent l'inspecteur général Pierre-JacquesNicolas Rolland, et le directeur des ports et arsenaux Jean-Marguerite Tupinier. Ces deux grandes figures du génie maritime, titulaires pendant plus de vingt ans des postes les plus importants du ministère de la Marine, sauront, malgré les vicissitudes du pouvoir, donner l'impulsion nécessaire à un renouveau de la marine. Ils peuvent être considérés comme les pères fondateurs des écoles de maistrance. Le chemin parcouru pendant les 150 années qu'embrasse notre étude a été très long et semé d'embûches. Les premières écoles de maistrance, comme les écoles des arts et métiers ou le conservatoire des arts et métiers, avaient pour but de former des ouvriers habiles et possédant un minimum d'instruction. Elles semblent avoir dès le début atteint leur objectif, contrairement aux premières tentatives, vite abandonnées, de formation à l'école des arts et métiers de Châlons-sur-Marne dont l'enseignement ne répondait pas aux besoins spécifiques des constructions navales. L'économie a été pendant très longtemps la principale préoccupation des responsables de la marine, qui souhaitaient disposer d'une maistrance habile, sans vouloir y consacrer les moyens indispensables. La nécessité de donner à cette maistrance un bon niveau d'instruction était bien reconnue, mais pour certains ingénieurs de direction, craignant peut-être de voir s'instituer une certaine concurrence, il s'agissait d'une classe «qu'il est essentiel de tenir très modeste ".

30 Ce seront les circonstances difficiles de la Seconde Guerre mondiale et de l'occupation du pays qui entraîneront la naissance d'une véritable école, enfin regroupée en un même lieu. L'enseignement sera commun pour tous les élèves d'une même promotion, et surtout la direction sera composée d'ingénieurs qui n'ont plus d'autre fonction que celle de gérer les écoles techniques et d'en organiser l'enseignement, tout d'abord à Aurillac en 1943, puis à Brest en 1949. Cette unité de direction permettra de disposer 
enfin d'un outil de formation des cadres et des ingénieurs bien adapté aux besoins des constructions navales.

En sera-t-il de même à l'avenir? Nous nous permettons d'en douter, car les données de base ont été bouleversées. D'une part les profondes réformes du cycle d'enseignement ont d'abord nettement séparé le recrutement et la formation du personnel ouvrier de ceux des cadres techniques et ingénieurs, puis purement et simplement supprimé les écoles d'apprentis. D'autre part, le recrutement massif d'ingénieurs civils provenant d'écoles de tous niveaux, et disposant de statuts différents, s'est rapproché des conditions de l'industrie privée. Le récent remaniement de la direction des constructions navales, devenue société DCN, va largement dans ce sens et contribuera sans aucun doute à accélérer le phénomène. La formation pratique de base de la majorité des anciens élèves des écoles techniques et leur brève affectation dans les ateliers ou les bureaux d'études avant leur départ pour les écoles, leur donnaient auprès du personnel, ouvriers ou techniciens, issus pour la plupart de la même formation initiale et connaissant bien les difficultés de l'accès aux différents échelons, une certaine considération, favorable à des relations confiantes. Un système original de culture d'entreprise et de promotion sociale, que d'aucuns auront pu qualifier de paternaliste, ne sera bientôt plus qu'un souvenir.

\section{BIBLIOGRAPHIE}

Acerra, Martine

1993. Rochefort et la construction navale française (1661-1815). Thèse pour le doctorat, sous la direction de Monsieur le professeur Jean Meyer. Paris IV, Sorbonne, Paris, Librairie de l'Inde.

Berthiau, Jean André

1997. Des maîtres entretenus aux ingénieurs. Formation professionnelle et enseignement technique dans les arsenaux et établissements de la marine (1819-1971). Thèse pour le doctorat, sous la direction de Monsieur le professeur Jean Tulard,.Paris IV, Sorbonne.

1999. Des Maîtres entretenus aux ingénieurs 1819-1971. Paris : Service historique de la Marine.

L'enseignement technique dans la marine

1932. Plaquette du centenaire. Paris : AAETSM.

\section{NOTES}

1. Martine Acerra (1993) en donne une étude détaillée dans sa thèse.

2. Cet article est un bref résumé de la thèse soutenue par l'auteur (Berthiau 1997).

3. Ordonnance du 12 février 1834.

4. Décret du 7 avril 1851.

5. Décrets du 14 juin 1865.

6. Décret du 8 février 1868.

7. Décret du 6 mars 1877. 
8. Décret du 2 juillet 1881.

9. Décret du 12 juin 1877.

10. Décrets du 15 janvier 1900.

11. Décret du 25 janvier 1912.

12. Loi du 30 décembre 1913 sur les pensions.

13. Décret du 14 novembre 1925.

14. Décret du 11 août 1934.

15. Décret du 2 novembre 1945.

16. Arrêtés ministériels du 29 avril 1955.

\section{RÉSUMÉS}

En 1819, un enseignement spécifique est mis en place dans les ports pour les ouvriers destinés à la maistrance. Les changements techniques dans les constructions navales entraînent adaptations de programmes et réorganisations des écoles, notamment en 1868 avec l'instauration de deux niveaux d'enseignement. La création en 1934 du brevet d'ingénieur diplômé ETSM marque la reconnaissance de l'enseignement dispensé à l'école technique supérieure. Une véritable grande école voit le jour en 1943 avec une implantation unique à Aurillac, suivie de l'installation à Brest-Pontanézen en 1949. L'école technique supérieure laisse la place en 1971 à l'ENSIETA. Elle perd alors sa spécificité dans les constructions navales.

\section{From « sustained masters " to engineers.}

In 1819 , a specific education is set up in the harbours for the workers intended to the "maistrance». The technical changes in shipbuilding bring about adaptations in programs and re-organizations of the schools, notably in 1868 , with the setting up of two teaching levels. The creation, in 1934, of the ETSM certified engineer diploma marks the recognition of the teaching quality provided in the higher technical school. A real higher school is born with one single establishment in Aurillac in 1943, moving to Brest-Pontanezen in 1949. The higher technical school is replaced in 1971 by ENSIETA, thus losing its speciality of shipbuilding.

De maestros « sostenidos » a ingenieros (1819-1971).

En 1819 se instauró en los puertos de mar un sistema de enseñanza específico, dirigido a los obreros destinados a la maestranza. Los cambios técnicos que se habían producido en la construcción naval implicaban la necesidad de adaptar los programas de estudios y reorganizar las escuelas, especialmente a partir de 1868 en que se establecieron dos niveles de enseñanza. La creación en 1934 del diploma de ingeniero diplomado ETSM supuso el reconocimiento de un nivel de enseñanza equiparable a la escuela técnica superior. Una auténtica "grande école» (institución de enseñanza superior prestigiosa) vio la luz en 1943, primero en la sede de Aurillac, y más adelante con su establecimiento en Brest-Pontanézen en 1949. La escuela técnica superior dejó paso en 1971 a la ENSIETA, perdiendo su especificidad en las construcciones navales. 
INDEX

Mots-clés : constructions navales, écoles, ingénieurs, maître entretenu, marine

Keywords : shipbuilding, formation, schools, engineers, sustained masters, navy

\section{AUTEUR}

JEAN ANDRÉ BERTHIAU

Ingénieur en chef de 1ère classe des Études et Techniques d'Armement 